\title{
Design Of Automatic Test System For Transmitter Controller
}

\author{
Fangfang LIU, Hongshe Dang \\ Shaanxi University of Science \& Techonlogy, Xi'an Shaanxi 710021,China
}

Keywords: controller; automatic test; LabVIEW; TestStand

\begin{abstract}
In the transmitter, the controller is used to realize the transmitter real-time monitoring and control.In order to guarantee the quality of the transmitter controller, for manual testing, high repetition rate, low efficiency and low accuracy problem, a set of automatic test system based on LabVIEW and TestStand has been designed and developed.Mainly introduced the the design process of the automatic test and test results for transmitter controller function, the system can test the function of the controller quickly and accurately according to the established sequence, it can effectively improve the test efficiency of the staff.
\end{abstract}

\section{Introduction}

Transmitter controller as a broadcast transmitter control device, which can realize to broadcast transmitter automatically open, shut down, can real-time monitoring of the working point of transmitter, and displayed, can record the working state of the machine for a long time, record the fault in time and protect transmitter when faults occurred ${ }^{[1]}$. The stand or fall of controller directly affect the normal operation of the transmitter, if the controller cannot monitor the running state of the transmitter correctly, it cannot make the right judgment, also cannot implement the protective function. This can lead to the damage of the transmitter, and cause great financial losses.So the controller must be tested before installed in the transmitter, to ensure its correctness.

Currently, the transmitter controller test mainly manual testing, high repetition rate, low efficiency and the accuracy is low ${ }^{[2,3]}$, but the controller cannot be repeated tests directly installed on the transmitter, it can cause the damage of the transmitter.Besides, if the controller has faults, the staff can't find the problem quickly,after repairing,they cannot know whether the faults have been ruled out.so a kind of equipment can quickly and easily to test the validity of the controller is needed.we call such equipment as broadcast transmitter controller test platform.It is very convenient to test the correctness of the broadcast transmitter controller. When the broadcast transmitter controller number is more, its superiority show obviously, greatly improve the productivity, and in the later maintenance inspection, the test platform can be convenient to test after repair of controller.

So, this paper designed the automatic test system of transmitter controller to test controller, this system can automatically test, reduce the staff repeatedly testing state of boring, also can make the test more accurate, and provides guarantee for the quality of the controller before delivery, and provides a convenient maintenance and fault diagnosis in the later.

\section{The structure of the object and test requirement analysis}

Measured object system structure as shown in figure 1, designed by DCS structure.Main control board not only realize the control tasks, but also realize the human-computer interaction function, display the real-time status of transmitter. Power supply control board and power amplifier control panel is on the basis of mutual information to the master control board, independently completed on transmitter control tasks of each module.Collaboration between two levels, complete control of the transmitter of the whole system.

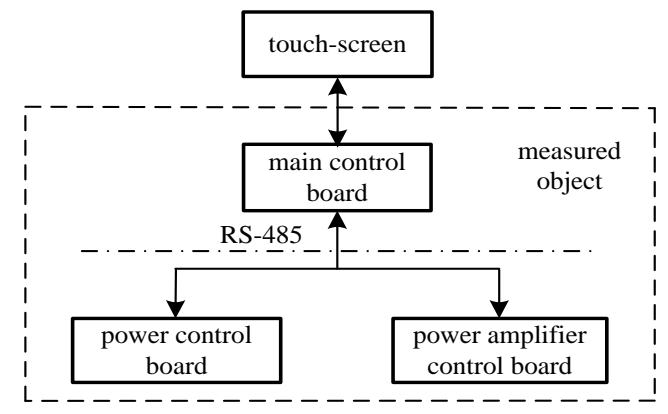

Figure 1: System structure of measured object

The specific function of requirements test system need to test are as follows.

1)Turn on/off function:achieve automatic and manual two kinds of open and shut down mode;

2)Monitoring function:real-time monitoring the running status of radio transmitter modules;

3)Data query functions:real time and history data query;

4)Parameter setting function:system parameters set and store; 
5)Fault detection and protection function:to detect the occurrence of failure and according to the different failure classification processing, record and save.

\section{The structure of the measured object and test requirement analysis}

Overall test principle of automatic test system is shown in figure $2^{[4,5,6]}$. The LabVIEW software and TestStand test management software on PC provide good human-machine interface to the operators,make test process control and processing. Data acquisition card is responsible for executing instructions from the computer, output analog and switch quantity, and transfer control commands to the computer come from the broadcast transmitter controller. Matrix switch is responsible for the signal of the gate. Interface adapter according to the measured controller design realizes the controller to be tested and interface characteristic of the connection between the data collection instruments.Store data and generate reports after the completion of the test.

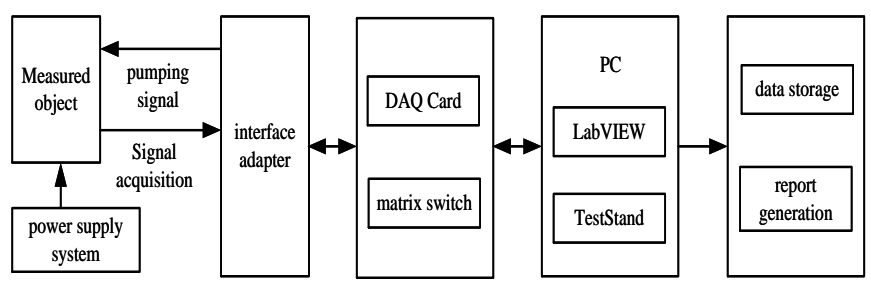

Figure 2: Overall diagram of the test system

\subsection{The hardware design of automatic test system}

The hardware of test system includes computer, DAQ card, matrix switch, interface adapter.Choose the data acquisition card NI USB-6211, it is a bus power supply USB M series multifunction DAQ module, can maintain high precision under the high sampling rate ${ }^{[7]}$.The plug and play installation to reduce the configuration and setup time.Interface adapter according to the measured object interface design, Power supply control board and power amplifier panel each have three DB15 and one 485 interface, so, the schematic diagram is shown in figure 3 .

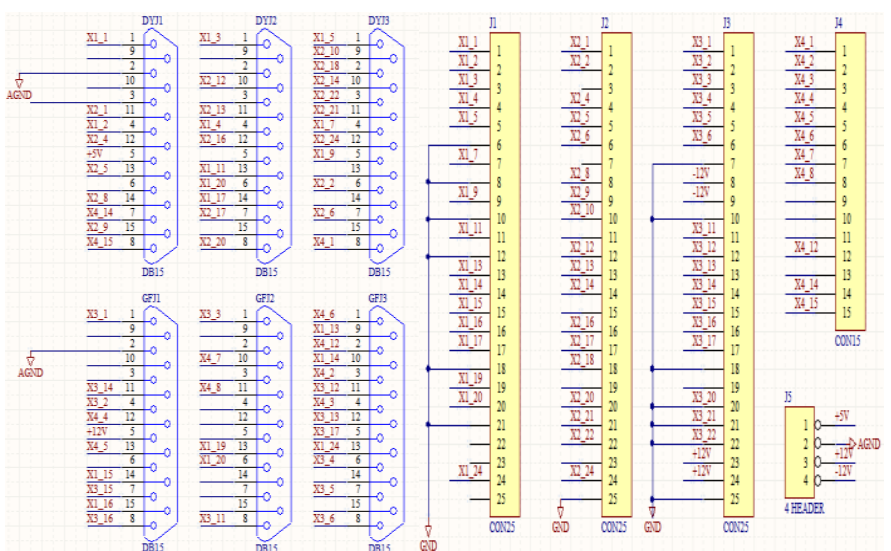

Figure 3: Principle diagram of the interface adapter

\subsection{The software design of automatic test system}

Test system software mainly function is to output analog signals and on-off signal that measured object needed, receive switch signals from the measured object, and can simulate the fault signal, can realize signal acquisition and control, signal analysis and processing, the expression of test results and output, etc ${ }^{[8,9]}$.

In this paper, using labview and teststand complete test system software development.Software part adopts the modular, hierarchical design in order to realize the real-time data acquisition, processing, display, storage, and other functions. The software system structure diagram as shown in figure 4 , design of human-computer interaction interface automatic test in LabVIEW, the tester can operate easily and directly in the interface, can be directly to call designed programming module. In the TestStand test management software can be for the whole testing process management, test the measured object according to the set test sequence.

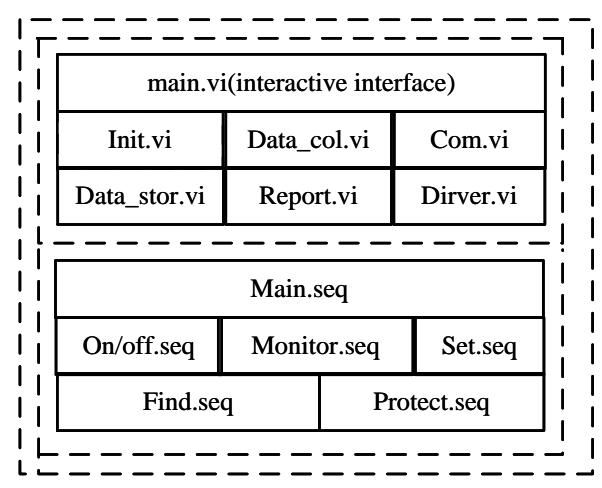

Figure 4: Structure diagram of software

Mainly introduces the test system of analog output module, the switch quantity acquisition module and data processing module, the program block diagram is shown in figure 5 .

1) The analog output module

DAQ hardware configuration and test of system by using MAX.System hardware testing through the DAQ assistant, Terminal configuration, sampling mode, sampling channel, sampling rate, sampling frequency and the input signal range settings, output corresponding analog to the controller by using data acquisition card combined with software.

2) the switch quantity acquisition module

Before the task to create a DAQ, first initialize the equipment. Mesurement \& Automention Explorer is used to Initialize the equipment.The NI- DAQmx task can create after the device initialization . Set the appropriate sampling parameters, collect controlled quantity feedback from measured controller.

3) data processing module

Comparing the collected signal and theoretical value, output result and generate reports.In order to in-depth analysis 
follow-up on the system data collected, this module has realtime data file saved and results display, and other functions.

\subsection{The design of test system process}

Process design of automatic test system is shown in figure 5. First, initialize system hardware and parameter, to ensure test accurately and effectively. Then read the test configuration file.Test system output corresponding excitation signal to the measured controller.Measured Controller make the response after receiving the signal, and the response signal is fed back to the test system. Test signal processing module will receive the signal and compared with the theoretical value.Comparing the measured values are consistent with the theoretical value, if the response error within the scope of the permit, then the test to pass, if it is beyond the scope of the error, prompt test failed, display an error message.Exit is if the test is over, if is not over, according to the above test steps to continue testing.

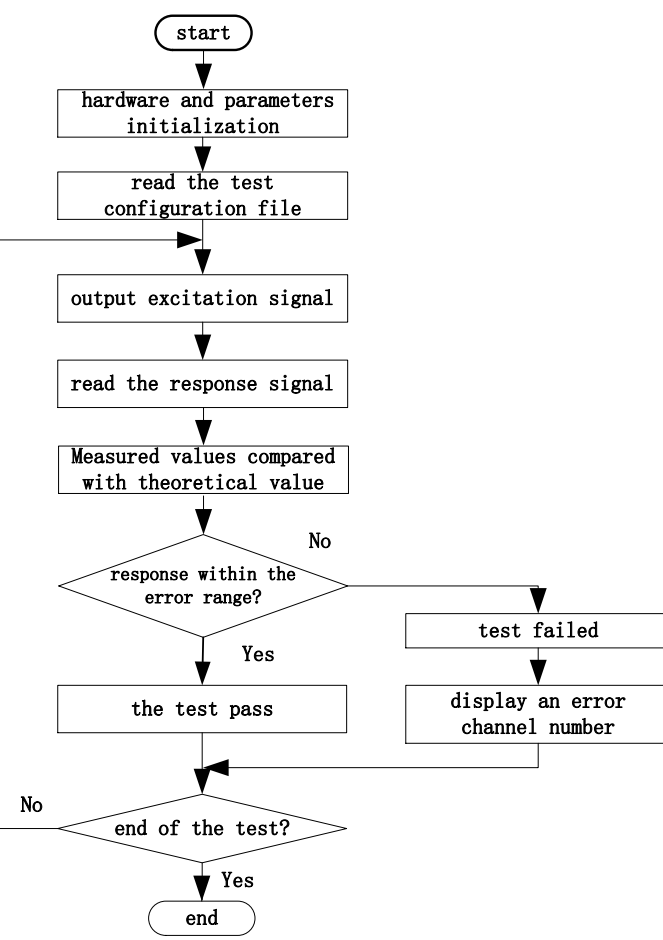

Figure 5: Flowchart of controller test

\section{Test implementation}

Physical test system diagram as shown in figure 6 , according to the test sequence to test it.

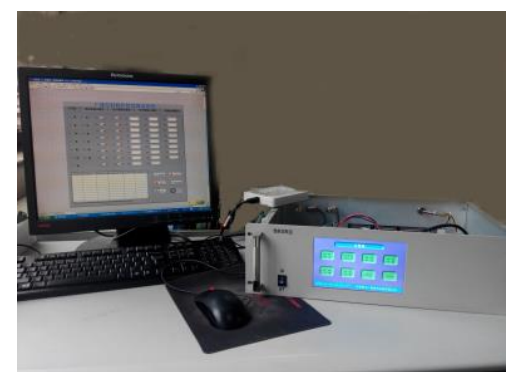

Figure 6: Picture of controller test device
To test the whole function of transmitter controller, because there is a definite relation between controller function, such as fault handling function test needs under the premise of success in the real-time data acquisition test. So need to formulate a reasonable order for testing. The controller function test order is test the controller turn on/off function first, followed by the real-time data acquisition and display function, and then test the fault handling function.

Firstly, test the turn on/off function. This test divided into sequential testing and troubleshooting test.Sequential testing corresponding state is set to normal signal, test if controller can turn on/off transmitter smoothly, then sets the signal to abnormal, test whether controller can give tips and operating correctly.Turn on transmitter involves fan, high pressure, driver and so on.Only when each state is normal can be switched on.

Secondly, test the real-time data acquisition and display function.Before the test, set up corresponding parameters, such as sampling rate, channel, etc.Main control board need to set into a "boot" machine condition.Start the master control unit polling data task of each control unit and blocking the control of the main control unit protection routine.In interactive interface input corresponding analog and switch quantity, then the touch screen receives data and shows the results on a touch screen, the picture as shown in figure 7.The test results show that the transmitter controller can collect and display data correctly.

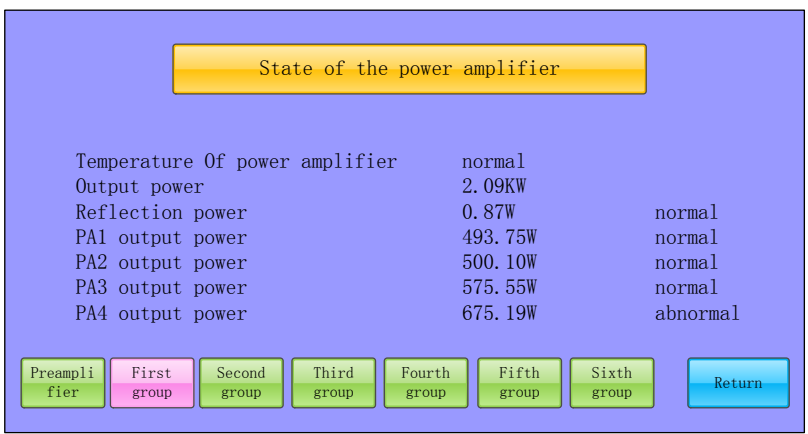

Figure 7: Data acquisition and display test result

Thirdly, test the fault handling function.Transmitter controller has three methods to handle the fault according fault degree. When controller receives the signal from transmitter, if any one of three types of fault is detected, controller should make protect action according to the fault handling regulations. When there are several types of fault at the same time, handle the fault according to the most severe type of fault processing method(fault handling priority: class III > class II >class I). So as long as test system can simulate the three kinds of failure, it can test if the controller fault protection function is correct.

\section{Conclusion}

In this paper, measured controller automatic test system is developed based on the LabVIEW and NI TestStand software 
system. This system have these advantage. Firstly, this test system can complete test task that in the laboratory can't complete, reduce the rate of the transmitter damage.Secondly, simulation signal module and test module can be extended to the functional test of different power controller.Thirdly, in the transmitter controller later maintenance, when troubleshooting, you can directly use the test system to test transmitter controller,it provides great convenience for the transmitter controller maintenance.

\section{Acknowledgements}

Thanks for the support from tutor and shaanxi province department of science and technology research plan project funds. So that I can complete this paper smoothly, tanks again!

\section{References}

[1] Liu Jing, Dang Hongshe, Liu Xingliang, et al. "Design and Realization of Digital TV Transmitter Controller Communication System", Video Engineering, 35, pp. 46-47, (2011).

[2] Zhao Chengyong, Zhang Baoshun, Liu Qijian, et al. "Design and test of MMC sub-module controller", Electrical Measurement \& Instrumentation , 52, pp. 91-96, (2015).

[3] Wang Zhongyuan, Shang Yonghe, Liu Jiarui, et al. "A LabVIEW based automatic test system for sieving chips” , Measurement, 46, pp. 402-410, (2012). this test system can simulate the transmitter runtime state, greatly save time, improve the test efficiency, after the perfect

[4] Toku, H.Alper. “Developing new Automatic Test Equipments(ATE) using systematic design approaches" [C] // AUTOTESTCON, 2013 IEEE. IEEE, 2013:1-7.

[5] Shang Yonghe, Luo Hao, Yu Faxin, et al. "An efficient testing system and calibration method for a reflection type vector modulator", Measurement, 46, pp. 38023807, (2013).

[6] Kandalaft N, Attaran A, Rashizadeh R. "High speed test interface module using MEMS technology", Microelectronics Reliability, 55, pp. 374-382, (2015).

[7] Chen Xinxin, Xiao Chengdi, Li Junhui, et al. "Automatic alignment and testing system for wafer with ball grid array", Optik-International Journal for Light and Electron Optics, 127, pp. 4656-4660, (2016).

[8] Chen Anyu, Zhao Guoqing, Feng Huizong, et al. "Functional test method for electronic control unit based on controller area network bus", Journal of Computer Applications, 32, pp. 139-142, (2012).

[9] Petrenko A, Dury A, Ramesh S, et al. "A method and tool for test optimization for automotive controllers" $[\mathrm{C}] / /$ Software Testing, Verification and Validation Workshops(ICSTW), 2013 IEEE Sixth International Conference on. IEEE, 2013: 198-207. 